\title{
Distribution of Bax protein in the rat hippocampus
}

\author{
Pavel D. Lisachev \\ Institute of Computational Technologies SB RAS \\ Novosibirsk, Russia \\ lisachev@ngs.ru
}

\author{
Anna L. Proskura \\ Institute of Computational Technologies SB RAS \\ Novosibirsk, Russia \\ annleop@mail.ru
}

\begin{abstract}
Bcl-2 family protein Bax is involved in mechanisms of synaptic plasticity. The induction of long term potentiation in hippocampal slices leads to increased Bax expression, but the localization of these changes remains unclear. Bax immunoreactivity is visually detected mainly in the layer of pyramidal neurons and is rarely detected in S100Bpositive glial cells. However, a quantitative assessment of Bax colocalization with glial and neuronal markers (S100B and NeuN, respectively) showed that although the Bax content in S100B-positive glial cells is really low, the Bax neuronal somatic pool is not the main source of Bax protein in the hippocampus.
\end{abstract}

\section{Keywords - hippocampus; Bax; synaptic plasticity}

\section{Introduction}

Bcl-2 family proteins are the regulators of apoptosis, but also have other functions. The Bax protein belonging to this family forms a channel in the mitochondrial membrane through which, under stressful conditions, cytochrome c exits mitochondria to trigger activation of a cascade of proteolytic enzymes - caspases, which play, in particular, a key role in apoptosis [1]. This mechanism is also involved in the formation of glutamate N-methyl-D-aspartate (NMDA) receptor-dependent synaptic depression in hippocampal CA1 field. The low frequency $(1 \mathrm{~Hz})$ activation of the Schaffer collaterals leads to the limited activation of caspases mediated by the Bax protein, which ultimately leads to the internalization of glutamate $\alpha$-amino-3-hydroxy-5-methyl-4isoxazolepropionic acid (AMPA) receptors and, thereby, to a weakening of synaptic efficiency [2]. Though, during the formation of long term potentiation in the synapses of the Schaffer collaterals on the pyramidal neurons of the hippocampal CA1 field, an increase in Bax expression in the CA1 field homogenates is observed [3]. If this increase occurs in neurons, this may indicate that the long term potentiation of synaptic connections is accompanied by the activation of negative feedbacks, creating conditions for limiting growth or increasing a predisposition to reduce the effectiveness of glutamatergic synapses.

A preliminary analysis of the distribution of Bax in the rat hippocampus showed that Bax immunoreactivity is indeed visually detected mainly in the layer of pyramidal neurons and is rarely detected in S100B-positive glial cells [4]. In the present work, a more detailed quantitative assessment of Bax colocalization with glial and neuronal markers (S100B and $\mathrm{NeuN}$, respectively) was carried out.

\section{Methods}

Male Wistar rats (7-9 weeks old) were used in the experiments. After decapitation of the animal, the brain was placed in cold aerated saline. Immediately after isolation, fragments of the hippocampus were placed in $4 \%$ paraform. Primary anti-S100B and anti-NeuN antibodies from mouse and anti-Bax from rabbit, secondary goat anti-mouse IgGFITC and anti-rabbit IgG-DyLight594 were used. After immunohistochemical processing, hippocampal slices (20 $\mu \mathrm{m})$ were examined using a laser scanning microscope LSM $780, \times 20$ lens, $x / y$ coordinate resolution $0.83 \mu \mathrm{m} /$ pixel. When analyzing Bax colocalization with cell markers, in each histological slice, the data obtained on the four ( $2 \mu \mathrm{m}$ interval $)$ most representative optical sections were summarized. As a minimum estimate of the background fluorescence level, a MIN value was taken such that at least $99.99 \%$ of the voxels in the scan area outside the sample had a fluorescence level less than MIN. The colocalization coefficients of Bax with cell markers were calculated at threshold Bax levels Ln, which were determined as follows:

$$
L n=M I N+n(M A X-M I N) / 8
$$

Here $\mathrm{n}=0,1, \ldots, 7$, and MAX is such that no more than $0.1 \%$ of all voxels in the scan area had a fluorescence level greater than MAX. Average values are presented as "mean \pm standard deviation".

\section{Results}

The colocalization coefficient of Bax (the ratio of the number of colocalized voxels to the total number of Baxpositive voxels) with the glial cytoplasmic marker S100B, as a rule, did not depend on the threshold Bax level and ranged from 0.02 to 0.05 . This parameter reached its maximum value $(0.13)$ in one of six samples for voxels with a maximum level of Bax immunopositivity (at L7 threshold). In all cases, the colocalization coefficients were higher than the expected random values.

Neuron marker NeuN is expressed primarily in the nuclei of neurons. In smaller quantities, it was also present in the cytoplasm of somata and proximal dendrites. Although visually the degree of colocalization of Bax and the neuronal marker NeuN seems rather high, the coefficient of colocalization of Bax with NeuN turned out to be small - no more than 0.3. Moreover, it gradually increased with increasing threshold Bax level - from $0.06 \pm 0.02$ at L0 to 0.16 \pm 0.08 at L7. Obviously, the colocalization coefficient of Bax with NeuN underestimates the Bax content in neurons. Perhaps a significant part of Bax is located in the processes of cells, where it exists mainly in the form of small inclusions, which are hardly perceived visually. However, although the content of Bax in S100B-positive glial cells is low, it cannot be ruled out that a significant amount is present in cells of other types, for example, in blood vessels.

\section{Conclusion}

A visual analysis of the distribution of Bax in the hippocampal cells is insufficient to conclude that Bax is predominantly neuronal. To analyze cell specificity and neuronal activity-dependent dynamics of Bax expression, it may be appropriate to use animals with neuron-specific cytoplasmic expression of fluorescent markers. 


\section{Acknowledgment}

The histological preparations were examined at the Shared Centre for Microscopic Analysis of Biological Objects of the Institute of Cytology and Genetics SB RAS, the authors are thankful to Dr. S.I. Baiborodin for technical support.

\section{References}

[1] J. M. Hardwick and L. Soane, "Multiple functions of BCL-2 family proteins," Cold Spring Harb. Perspect. Biol., vol. 5, a008722, February 2013.
[2] Z. Li and M. Sheng, "Caspases in synaptic plasticity," Mol. Brain, vol. 5, 15, May 2012.

[3] V. O. Pustylnyak, P. D. Lisachev, M. B. Shtark, "Expression of p53 target genes in the early phase of long-term potentiation in the rat hippocampal CA1 area," Neural Plast., vol. 2015, 242158, 2015.

[4] P. D. Lisachev and M. B. Shtark, "Long-term potentiationassociated gene expression: involvement of the tumour protein p53," in The Hippocampus - Plasticity and Functions, A. Stuchlik, Ed. London: IntechOpen, 2018, pp. 49-64. 